\title{
A new species of Potentilla (Rosaceae): P. baekdusanensis M. Kim
}

\author{
Hyun JO, Changkeun SHIN, Eunkyoung SEO and Muyeol KIM*
}

Department of Biological Sciences, Chonbuk National University, Jeonju 54896, Korea

(Received 20 February 2018; Revised 15 March 2018; Accepted 17 March 2018)

\begin{abstract}
A new species, Potentilla baekdusanensis M. Kim, is described here. It is found in Baekdusan Mt., Korea. Potentilla baekdusanensis shares several characteristics (pinnately compound leaves, pubescent leaf blades and leaf petioles, different leaflets size, and absent stolons) with the related species $P$. fragarioides. However, this new species has small plants with short and curved hairs, 7 leaflets, small lower leaflets, a glabrous leaflet margin, and yellow petals with a reddish yellow base. In contrast, $P$. fragarioides are large plants with long and straight hairs, 9-15 leaflets, large lower leaflets, a ciliate leaf margin, and yellow petals without a reddish yellow base.
\end{abstract}

Keywords: Potentilla baekdusanensis, P. fragarioides, new species, Rosaceae, Baekdusan Mt.

The genus Potentilla L. (Rosaceae) consists of approximately 500 species and is distributed primarily in temperate, arctic, and alpine zones of the Northern Hemisphere (Li et al., 2003).

Potentilla are characterized by yellow petals longer than sepals, many stamens, more than 10 carpels, and a dry fruiting receptacle (Lee, 2007). Potentilla consists of 6 sections (Rhopalostylae, Nematostylae, Closterostylae, Leptostylae, Potentilla, Conostylae) in China and 4 sections (Rhopalostylae, Nematostylae, Potentilla, Conostylae) in Korea (Li et al., 2003). Potentilla fragarioides L. belongs to section Potentilla. Seventeen taxa of Potentilla are distributed in Korea (Lee, 2007). Of them, P. fragarioides L. and P. freyniana Bornm occur widely in Korea, whereas $P$. nivea L. and $P$. gageodoensis $\mathrm{M}$. Kim are rather rare and sporadically distributed (Lee, 2006; Heo et al., 2013; So et al., 2014; Kim, 2017).

A new species of Potentilla was discovered in Baekdusan Mt. in Korea. The new species shares several characters with its related species, $P$. fragarioides L. However, it can be distinguished from $P$. fragarioides based on plant size (i.e., small), leaflet hairs (i.e., short and curved), leaflet margin (i.e., glabrous), and petal base (i.e., reddish yellow). Thus, we named this new species as Potentilla baekdusanensis M. Kim.

\section{Taxonomic Treatment}

Potentilla baekdusanensis M. Kim, sp. nov. (Figs. 1-3). TYPE: KOREA. Baekdusan Mt., elev. 800 m, 23 Apr 2017. M. Kim MK176212 (holotype, JNU; isotypes, JNU, KH, KB).

Korean name: Baek-du-san-yang-ji-kkot 백두산양지꽃

Diagnosis: Potentilla baekdusanensis M. Kim can be distinguished from its related taxa $P$. fragarioides by having smaller plants with short and curved hairs, 7 leaflets, small lower leaflets, glabrous leaflet margin, and yellow petals with a reddish yellow base (Table 1).

Herbs perennial. Roots numerous. Stolons absent. Flowering stems 5-22, ascending or spreading, 7-11 cm in length. Basal leaves pinnately compound with 7 leaflets, 4$9 \mathrm{~cm}$ including petiole; stipules light-brown, abaxially sparsely spreading villous; leaflets at intervals of $0.5-1.0 \mathrm{~cm}$, shortly petiolulate or subsessile, pale green on abaxial surface, leaflets elliptic, $1.5-2.0 \times 1.0-1.5 \mathrm{~cm}$, adaxial surface pilose or not and abaxial surface pilose on vein, margins serrate and glabrous. Cauline leaves 2, 3-foliolate, leaflets obovate or elliptic, $0.9 \times 0.4 \mathrm{~cm}$, abaxial surface pilose, margin serrate and glabrous. Inflorescence terminal, corymbose-cymose. Flowers bisexual, $1.3-1.6 \mathrm{~cm}$ in diameter. Pedicel $1.9-2.5 \mathrm{~cm}$, with short and curved hairs. Sepals 5, ovate, 3.5-4.0 × 2.0-

\footnotetext{
*Author for correspondence: mykim@jbnu.ac.kr
} 
$2.5 \mathrm{~mm}$; epicalyx segments 5, lanceolate, $2.3-2.8 \times 1.2-$ $1.5 \mathrm{~mm}$, with short and curved hairs. Petals 5 , yellow with a reddish yellow base, circular, 6-7 × 6-7 mm, apex emarginate. Style 1, subterminal, clavate, thickened at apex, $1 \mathrm{~mm}$ in length. Fruits achene, seeds subreniform, $1.0 \times 0.8 \mathrm{~mm}$.
Flowering: April-May.

Distribution: Korea (Baekdusan Mt.).

Etymology: The specific epithet is derived from Baekdusan Mt., where this new species is found.

Habitats: A population of Potentilla baekdusanensis M.
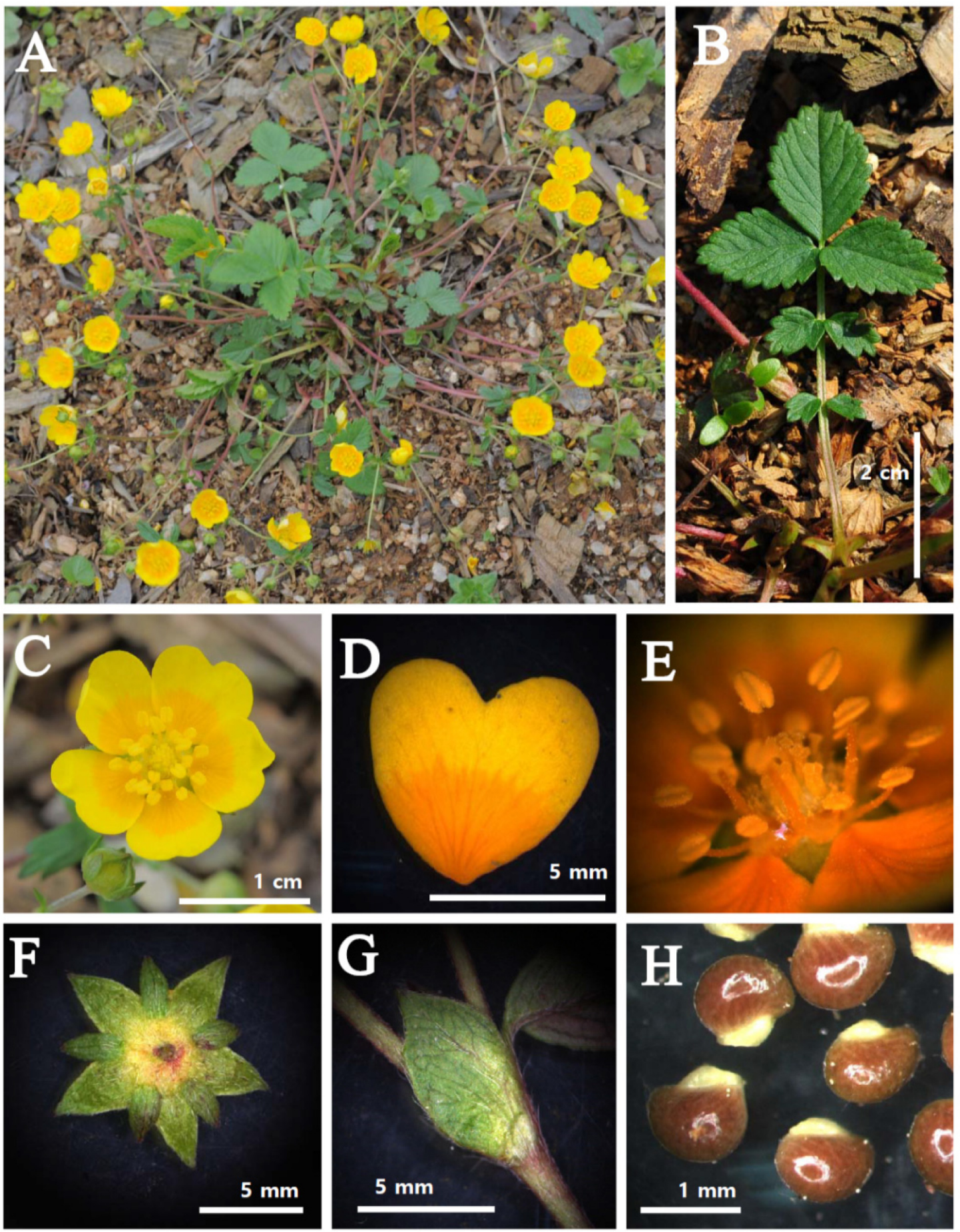

Fig. 1. Photographs of Potentilla baekdusanensis M. Kim. A. Adult plants with flowers. B. Leaf. C. Front view of flower. D. Petal. E. Pistils and stamens. F. Sepals and epicalyx segments. G. Stipule. H. Seed. 

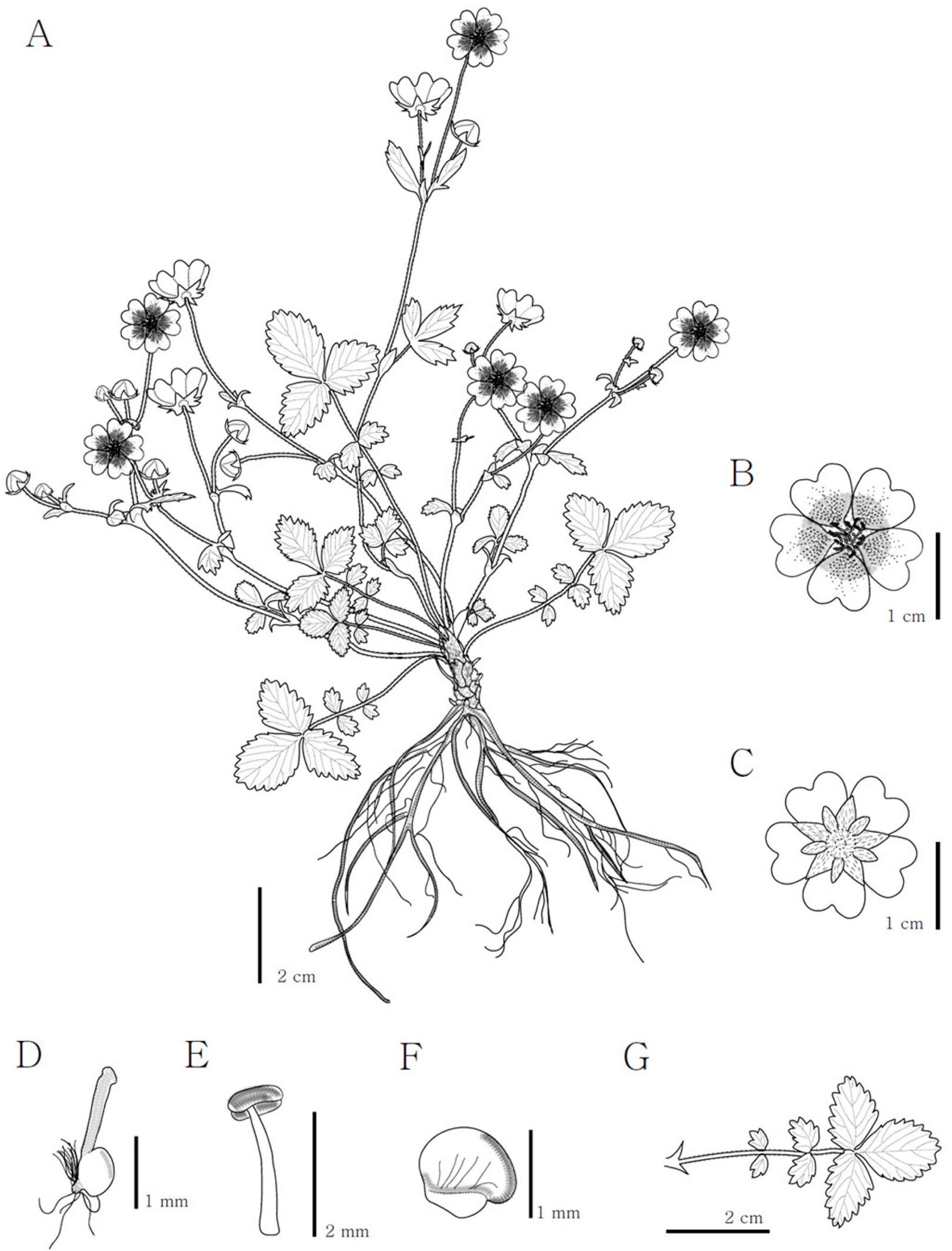

F

G

Fig. 2. Potentilla baekdusanensis M. Kim. A. Adult plant with flowers. B. Flower. C. Sepals and epicalyx segments. D. Pistil. E. Stamen. F. Seed. G. Basal leaf. 
Kim was found in woodlands on Baekdusan Mt.. This new species occurs under trees in forests dominated by Abies nephrolepis (Trautv.) Maxim. and Larix gmelinii var. olgensis (A. Henry) Ostenf. \& Syrach. This species also occurs with other herbaceous species such as Micranthes nelsoniana (D. Don) Small, Chrysosplenium flaviflorum Ohwi, Pyrola dahurica (Andres) Kom., and Lobelia sessilifolia Lamb.

Additional specimens examined (paratypes): KOREA. Baekdusan Mt., 19 May 2014. M. Kim MK140221 (JNU).

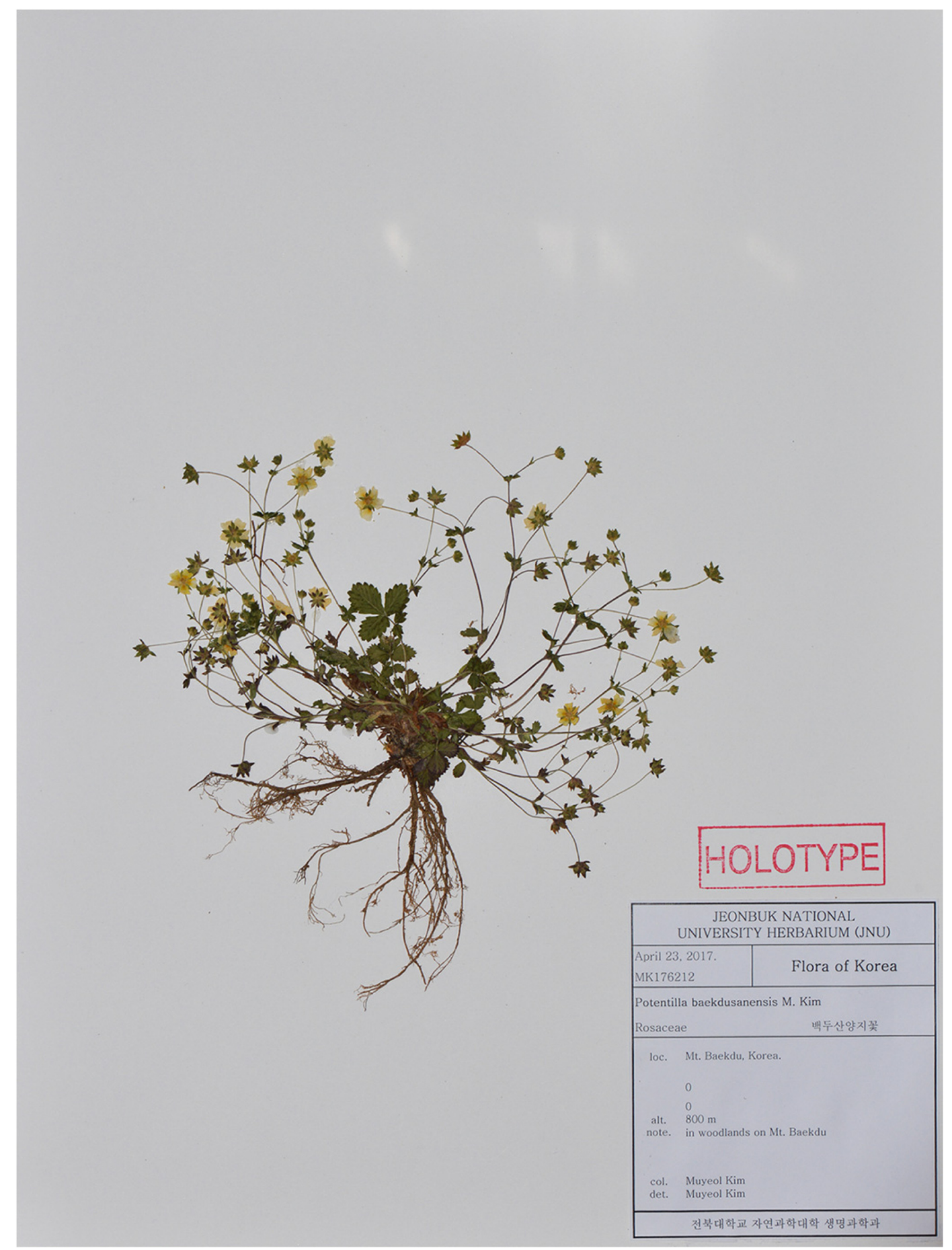

Fig. 3. Holotype of Potentilla baekdusanensis M. Kim. 
Table 1. Comparison of some morphological characters among Potentilla baekdusanensis and its related species.

\begin{tabular}{|c|c|c|c|}
\hline Characters & P. baekdusanensis & P. fragarioides & P. gageodoensis \\
\hline Plant size & Small & Large & Large \\
\hline Stolons & Absent & Absent & Absent \\
\hline Basal leaf length (cm) & $4-9$ & $20-30$ & $20-45$ \\
\hline Petiole color & Greenish purple & Greenish purple & Green \\
\hline Number of leaflets & 7 & $9-15$ & $5(7)$ \\
\hline Leaflet shape & Elliptic & Elliptic & Broadly ovate \\
\hline 3 Upper leaflets length $(\mathrm{cm})$ & $1.5-2.0$ & $2-6$ & $2.5-8$ \\
\hline 3 Upper leaflets width $(\mathrm{cm})$ & $1.0-1.5$ & $1-3$ & $2-6$ \\
\hline Lower leaflet length $(\mathrm{cm})$ & $0.3-0.5$ & $3-5$ & $2.5-3.5$ \\
\hline Leaflet margin & Glabrous & Ciliate & Densely white ciliate \\
\hline Number of leaflet teeth & $6-7$ & $9-11$ & $13-15$ \\
\hline Leaflet abaxial surface hairs & Pilose on vein & Densely pilose & Pilose on vein \\
\hline Leaflet abaxial surface color & Pale green & Pale green & Pale green \\
\hline Flowering stem length $(\mathrm{cm})$ & $7-11$ & $12-25$ & $19-30$ \\
\hline Flowering stem color & Greenish purple & Greenish purple & Green \\
\hline Number of cauline leaves & 2 & $3(5)$ & 3 \\
\hline Cauline leaf shape & Ovate & Elliptic & Ovate \\
\hline Number of flowers per peduncle & 6 & $11-12$ & $3-4$ \\
\hline Flower diameter $(\mathrm{cm})$ & $1.3-1.6$ & $1.3-2.0$ & $2.5-3.0$ \\
\hline Pedicel length $(\mathrm{cm})$ & $1.9-2.5$ & $1.5-2$ & $1-3$ \\
\hline Sepal shape & Ovate & Ovate & Lanceolate \\
\hline Sepal size $(\mathrm{mm})$ & $3.5-4.0 \times 2.0-2.5$ & $4-5 \times 1-2$ & $4.5-5.5 \times 1.5-2.5$ \\
\hline Epicalyx segment shape & Lanceolate & Lanceolate & Oblanceolate \\
\hline Epicalyx segment size (mm) & $2.3-2.8 \times 1.2-1.5$ & $4-5 \times 1-2$ & $4.8-5.3 \times 1.8-2.3$ \\
\hline Epicalyx hairs & Short and curved & Long and straight & Long and straight \\
\hline Petal color & Yellow with a reddish yellow base & Yellow & Yellow \\
\hline Petal length (mm) & $6-7$ & $5-7$ & $10-11$ \\
\hline Petal width (mm) & $6-7$ & $5-7$ & $10-11$ \\
\hline Seed shape & Subreniform & Subreniform & Subreniform \\
\hline
\end{tabular}

\section{Key to Potentilla baekdusanensis and its related taxa}

1. Style conic, thickened at base (Sect. Conostylae).

2. Leaves ternately compound …..P. nivea 은양지꽃

2. Leaves pinnately compound.

3. Leaflets lobed P. chinensis 딱지 꽃

3. Leaflets serrulate.

4. Stolons present …P. stolonifera 제주양지꽃

4. Stolons absent.
5. Leaflets lower surfaces glabrescent

P. supina 개소시랑개비

5. Leaflets lower surfaces pilose or glaucous . P. dickinsii 돌양지꽃

1. Style clavate, thickened at apex (Sect. Potentilla).

6. Stolons present; leaves 3-foliolate or palmately 5foliolate.

7. Leaves 3-foliolate ……P. freyniana 세잎양지꽃

7. Leaves palmately 5-foliolate P. flagellaris 덩굴뱀딸기 
6. Stolons absent; leaves pinnately compound.

8. Leaflets thin, elliptic, margin ciliate or glabrous; petals $0.6-0.7 \mathrm{~cm}$ long.

9. Basal leaves 4-9 cm; leaflets 7, lower ones small $(0.4-0.6 \mathrm{~cm})$, margin glabrous; petal base reddish yellow

P. baekdusanensis 백두산양지꽃

9. Basal leaves 20-30 cm; leaflets 9-15, lower ones large (1-3 cm), margin ciliate; petal base yellow P. fragarioides 양지꽃

8. Leaflets thick, broadly ovate, margin densely white ciliate; petals $0.8-1.1 \mathrm{~cm}$ long

P. gageodoensis 가거양지꽃

By applying the IUCN Red List criteria (IUCN, 2011), Potentilla baekdusanensis $\mathrm{M}$. Kim will be evaluated as the category of endangered (EN) species, because of its small population of five hundred individuals found on Baekdusan Mt..

\section{Conflict of Interest}

Authors declare that there is no conflict of interest.

\section{Acknowledgments}

We are deeply indebted to two reviewers for his kind help.

\section{Literature Cited}

Heo, K.-I., S. Lee, M. Yoo, S. Lee, Y. Kwon, S. Y. Lim, S. Kim and S.-C. Kim. 2013. The taxonomical implication of trichome and epicuticular waxes in tribe Potentilleae (Rosaceae) in Korea. Korean Journal of Plant Taxonomy 43: 106-117. (in Korean)

IUCN. 2011. Guidelines for Using the IUCN Red List Categories and Criteria. Version 9.0. Retrieved Jun. 10, 2014, available from http://www.iucnredlist.org.

Kim, M. 2017. Korean Endemic Plants. Haejin Media Co., Seoul. Pp. 197-198. (in Korean)

Lee, S. 2007. Potentilla L. In The Genera of Vascular Plants of Korea. Park, C. W. (ed.), Academy Publishing Co., Seoul. Pp. $549-553$

Lee, Y.-N. 2006. New Flora of Korea. Vol. I. Kyohak Publishing Co., Ltd., Seoul, Korea. Pp. 535-543. (in Korean)

Li, C., H. Ikeda and H. Ohba. 2003. Potentilla L. In Flora of China. Vol. 9. Pittosporaceae through Connaraceae. Wu, Z. Y., P. H. Raven and D. Y. Hong (eds.), Science Press, Beijing and Missouri Botanical Garden Press, St. Louis, MO. Pp. 291327.

So, S., H. Jo and M. Kim. 2014. A new species of Potentilla (Rosaceae): P. gageodoensis M. Kim. Korean Journal of Plant Taxonomy 44: 175-177.

\title{
양지꽃속(장미과)의 신종: \\ 백두산양지꽃(Potentilla baekdusanensis M. Kim)
}

\author{
조 현 · 신창근 · 서은경 · 김무열* \\ 전북대학교 자연과학대학 생명과학과
}

적 요: 백두산에서 양지꽃속의 신종인 백두산양지꽃(Potentilla baekdusanensis M. Kim)을 새로이 발견하여 기재하였다. 백두산양지꽃은 잎이 우상복엽이며 엽신과 엽병에 털이 있고 3 개의 상부 소엽이 하부 소엽보다 크며 포복경이 없어 양지꽃과 유사하다. 그러나 백두산양지꽃은 식물체가 소형이고 짧은 누운 털이 있으며 근생엽이 7장의 소엽으로 이루어졌고 하부 소엽이 작으며 소엽가장자리에 털이 없고 꽃잎 기부에 황적색의 무늬가 있다. 반면에, 양지꽃은 식물체가 대형이고 긴 곧은 털이 밀생하며 근생엽이 9-15장의 소엽으로 이루 어졌고 하부 소엽이 크며 소엽가장자리에 긴털이 밀생하고 꽃잎 기부에 황적색의 무늬가 없는 점에서 두 종 이 뚜렷하게 차이가 난다.

주요어: 백두산양지꽃, 양지꽃, 신종, 장미과, 백두산 